\title{
MODELOS DE CRECIMIENTO EN ALTURA DOMINANTE PARA Eucalyptus grandis Hill ex Maiden y E. urophylla S. T. Blake EN OAXACA, MÉXICO
}

\section{DOMINANT HEIGHT GROWTH MODELS FOR Eucalyptus grandis Hill ex Maiden and E. urophylla S. T. Blake IN OAXACA, MÉXICO}

\author{
Joaquín Gómez-Tejero ${ }^{1}$, Héctor M. De los Santos-Posadas*2, Aurelio M. Fierros-González² y J. René \\ Valdez-Lazalde ${ }^{2}$
}

\begin{abstract}
${ }^{1}$ Red de Plantaciones Forestales, Campo Experimental Edzná, Instituto Nacional de Investigación Forestales, Agricolas y Pecuarias. Km. 17.5 Carretera Cayal-Edzná. 24570, Usahzil-Edzná, Campeche. ${ }^{2}$ Postgrado Forestal, Colegio de Postgraduados. Km 36.5 Carretera México-Texcoco. 56230, Montecillo, Texcoco, Estado de México. Tel (595) 952-0200 ext 1484.
\end{abstract}

*Autor para correspondencia (hmsantos@colpos.mx)

\section{RESUMEN}

Un grupo de familias de curvas de índice de sitio basados en los modelos de Schumacher y Hossfeld IV se ajustaron con mínimos cuadrados ordinarios a datos de plantaciones de Eucalyptus grandis y $E$. urophylla de rápido crecimiento en el sureste de México. Con datos de la primera remedición se validaron las proyecciones de las familias de índice de sitio obtenidas a partir de Schumacher y Hossfeld IV. El crecimiento en altura dominante para $E$. grandis se estimó más eficientemente con la familia anamórfica derivada de Hossfeld IV, mientras que para $E$. urophylla se estimaron mejor con la familia polimórfica 1 de Hossfeld IV.

Palabras clave: Eucalyptus grandis, E. urophylla, índice de sitio, Schumacher, Hossfeld IV.

\section{SUMMARY}

A series of site index families based on the Schumacher and Hossfeld IV models were fitted through ordinary least squares on data from fast-growing plantations of Eucalyptus grandis and $E$. urophylla on southeastern México. Data from the first remeasurement were used to validate dominant-height predictions based on the several site index families derived from Schumacher and Hossfeld IV. For $\boldsymbol{E}$. grandis the dominant height predictions based on the anamorphic Hossfeld IV family proved to be the most efficient, meanwhile for $E$. urophylla the Hossfeld IV polymorphic 1 proved to be the best predicting family.

Index words: Eucalyptus grandis, E. urophylla, site index, Schumacher, Hossfeld IV.

\section{INTRODUCCIÓN}

La determinación de la familia de curvas que mejor describa los patrones de crecimiento en altura dominante de árboles es fundamental para integrar un sistema de crecimiento y rendimiento maderable (SCRM). El patrón de crecimiento en altura dominante de una masa forestal se usa tradicionalmente como indicador de la productividad total del sitio forestal. Así, los componentes de un SCRM, generalmente representados por el área basal, mortalidad y volumen maderable, están correlacionados con la altura dominante y sus proyecciones son afectadas por su patrón de crecimiento (Borders, 1989; Wang et al., 2007).

Los patrones de crecimiento se usan además para evaluar de manera cuantitativa y simplificada al conjunto de factores bióticos y abióticos que influyen sobre la productividad, y para determinar la calidad de estación. El sitio forestal puede clasificarse en zonas equiproductivas evaluadas a través de su altura dominante; a estas zonas se les asigna un valor en función de una altura dominante y una edad de referencia (edad base), y a este valor se le denomina índice de sitio (IS) (Cieszewski y Bailey, 2000).

Un modelo de IS representa una igualdad matemática de la forma $A_{p}=g\left(E_{I}, E_{p}, A_{I} ; p\right)$ donde: $A_{p}$ es la altura dominante a la edad $E_{p}$, a partir de la condición inicial de altura dominante $A_{I}$ a una edad de referencia $E_{I} ; p$ es el vector de parámetros; y $g($.$) es la función que caracteriza$ a la familia de curvas de IS. Cualquier estructura de este tipo representa una hipótesis específica sobre el patrón de crecimiento en altura dominante y refleja la productividad total del sitio forestal. El modelo permite no sólo estimar el crecimiento en altura dominante sino también caracterizar el sitio con su correspondiente IS cuando 
$A_{p}=I S$ y $E_{p}=E_{b}$, donde $E_{b}$ es la edad de referencia, más comúnmente llamada edad base.

Para obtener un modelo de la forma $A_{p}=g\left(E_{I}, E_{p}, A_{I} ; p\right)$ se parte de un modelo promedio de forma $A=f(E ; p)$ donde: $A$ es la altura promedio a la edad $E$ caracterizada por la función de crecimiento $f($.) y el vector de parámetros $p$. A partir de $A=f(E ; p)$ es posible obtener un modelo de la forma $A_{2}=g\left(E_{1}, E_{2}, A_{1} ; p^{(g)}\right)$ conocido como modelo de diferencia algebraica (DA) donde $p^{(g)}$ (subconjunto del vector de parámetros $p$ ) representa a los parámetros globales no específicos de sitio (Amaro et al., 1998; Cieszewski, 2003). Bajo la DA el número de familias de IS posibles es igual al número total de parámetros del modelo promedio. Cada familia representa además una hipótesis de crecimiento específica del sitio. Así, un modelo promedio de tres parámetros puede producir tres posibles familias de IS. Las posibles hipótesis de crecimiento que describe la función $g($.) representan un patrón de crecimiento donde la altura dominante máxima es específica al sitio y con una tasa constante de crecimiento (familias con múltiples asíntotas también conocidas como curvas anamórficas), o donde las tasas de crecimiento son variables entre sitios forestales pero con potencialidad máxima constante (curvas polimórficas).

Cuando se cuenta con datos provenientes de remediciones (mediciones consecutivas en un mismo sitio de muestreo forestal) es posible usar modelos de la forma $A_{2}=g\left(E_{1}, E_{2}, A_{1} ; p^{(g)}\right)$ y $A_{1}=f\left(E_{1} ; p\right)$ y ajustarlos simultáneamente para lograr compatibilidad entre los parámetros de la curva de proyección y predicción. La bondad de ajuste total del sistema se usa para la selección más adecuada de la familia de IS (Borders, 1989). Cuando se dispone únicamente de una medición puntual sólo es posible el ajuste de la curva promedio (conocida también como curva guía) y los valores estimados del vector $p$ se usan para proyectar el crecimiento con alguno de los modelos obtenidos con la DA.

Este estudio tuvo como objetivo ajustar un grupo de modelos promedio, a través de mínimos cuadrados ordinarios, y seleccionar la mejor familia de curvas de IS para plantaciones de rápido crecimiento de Eucalyptus grandis y E. urophylla a través de un esquema de validación. El proceso de validación evalúa la capacidad de los modelos de DA para predecir la altura dominante mediante el uso de datos de sitios con remediciones.

\section{MATERIALES Y MÉTODOS}

\section{Descripción del área de estudio}

La información utilizada para este estudio provino de Plantaciones Tehuantepec, S. A de C. V. (PLANTEH), empresa localizada en los límites de Veracruz y Oaxaca, en México, que desde 1997 ha establecido plantaciones forestales comerciales para producir madera para celulosa y aserrío, con un proyecto potencial de más de 20000 ha. En 2004 la empresa contaba con una superficie total plantada de 1717 ha, y de éstas 807 ha corresponden a Eucaliptus urophylla y las restantes 910 ha a E. grandis. La superficie total se encuentra distribuida en 13 predios bajo un contrato por 30 años; en estos predios se distribuyen ambas especies con edades de 12 a 90 meses (Telles et al., 2008)

El sitio de estudio se localiza en varios municipios de los Estados de Oaxaca y Veracruz, pero es en el municipio de Santiago Yaveo, Oaxaca, en donde hay la mayor superficie de plantaciones establecidas entre las coordenadas geográficas de $17^{\circ} 7^{\prime}$ a $17^{\circ} 54^{\prime}$ de $\mathrm{LN}$ y 94 '52' a $95^{\circ} 51^{\prime}$ LO. Los climas predominantes en la región son cálido húmedo $(\mathrm{Am})$ y cálido subhúmedo $(\mathrm{Aw})$, con temperaturas medias anuales que oscilan de 26 a $28{ }^{\circ} \mathrm{C}$ y lluvias que varían desde 1500 a $2400 \mathrm{~mm}$ (García, 1988). Sus suelos son Cambisoles, Alisoles y Luvisoles, según la clasificación de la FAO/UNESCO (1970), con textura franca o arcillosa de profundidad variable; su $\mathrm{pH}$ varía de 4.8 a 5.9 , por lo que son ligeramente ácidos. La topografía es poco accidentada y con una altitud promedio de $200 \mathrm{~m}$; la región se caracteriza por la presencia de planicies y lomeríos poco pronunciados. En la región predominan las áreas dedicadas a la ganadería extensiva de bovinos, y en algunos de esos terrenos (pastizales inducidos) se establecieron las plantaciones forestales analizadas en el estudio.

\section{Diseño de muestreo y toma de datos}

En la superficie plantada por la empresa (1717 ha) se establecieron 154 parcelas permanentes de muestreo, bajo un diseño de muestreo sistemático estratificado por especie y edad, para registrar los datos dasométricos. Las parcelas son de forma circular, cubren una superficie de $500 \mathrm{~m}^{2}$ (12.62 m de radio), y se establecieron en cuadrículas de 315 x 315 m de separación como mínimo entre sitios de muestreo. La intensidad de muestreo fue de $0.05 \%$.

En cada parcela se midió el diámetro normal de todos los árboles y la altura total de $20 \%$ de ellos, según la distribución diamétrica que incluye la de cinco árboles dominantes; a estos árboles también se les midió la altura 
de fuste limpio y la proyección de la copa. Esta primera medición se hizo de octubre del 2004 a junio del 2005.

Las parcelas se localizaron en una cronosecuencia, por lo que los datos permiten ajustar modelos de crecimiento para altura dominante. Para contar con datos que permitieran la validación de los modelos de crecimiento en altura dominante se hizo una remedición en 47 de las 154 parcelas permanentes, en el mes de febrero de 2006. Los datos de cada especie se muestran en la Figura 1 donde se aprecia que las remediciones se concentraron en las plantaciones de mayor edad que estaban cerca de la fase asintótica de crecimiento. De esta forma se buscó favorecer una selección de familias de IS con proyecciones conservadoras a edades mayores, etapa en la que puede haber sobreestimaciones considerables en el volumen maderable una vez que se integren los modelos de altura dominante al SCRM.

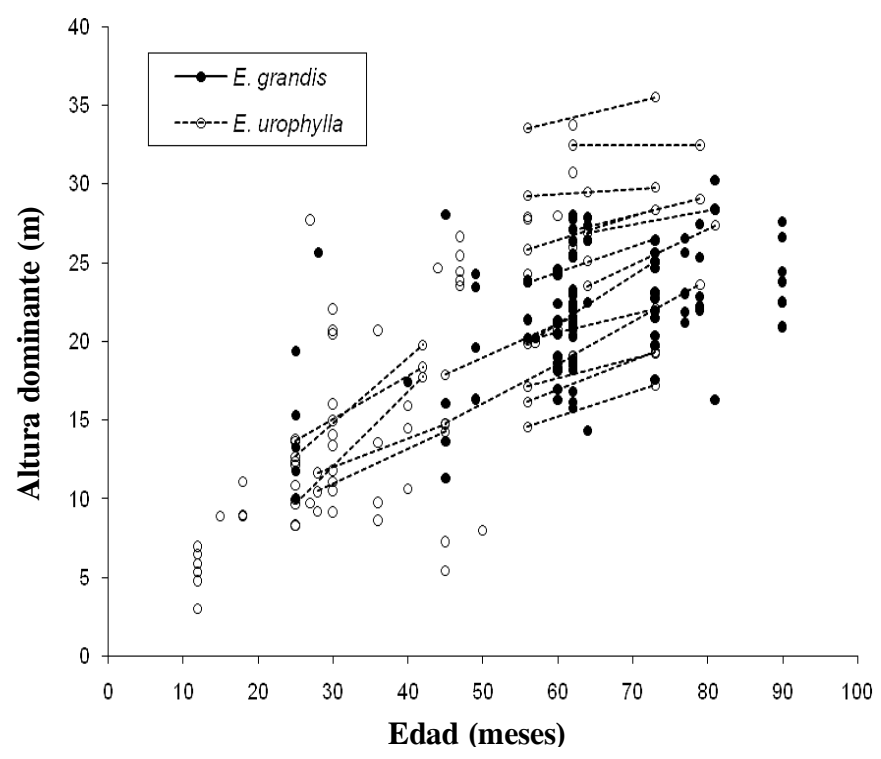

Figura 1. Datos de altura dominante por especie; los pares de datos unidos por líneas representan las parcelas donde se cuenta con remediciones.

\section{Familias de curvas}

Como base para el desarrollo de los modelos de IS se usaron los modelos promedio de Schumacher y Hossfeld IV. La nomenclatura y notación para el modelo de Schumacher (Schumacher, 1939; Zeide, 1993) es:

$$
A=\beta_{0} \times \exp \left(-\frac{\beta_{1}}{E}\right)
$$

Donde: $A$ es la altura dominante en metros; $E$ es la edad en meses; $\beta=\left(\beta_{0}, \beta_{1}\right)^{T}$ es el vector de parámetros a estimar. El modelo de Hossfeld IV (Cieszewski, 2001; Kiviste et al., 2002) es:

$$
\begin{aligned}
& A=\alpha_{0} \times\left(1+\exp \left(\alpha_{1}\right) \times \exp \left(-\alpha_{2} \times \ln (E)\right)\right)^{-1} \\
& =\alpha_{0} \times\left(1+\exp \left(\alpha_{1}\right) \times E^{-\alpha_{2}}\right)^{-1}
\end{aligned}
$$

Donde $\alpha=\left(\alpha_{0}, \alpha_{1}, \alpha_{2}\right)^{T}$ es el vector de parámetros a estimar, las variables restantes ya fueron definidas en la Ec. 1.

Los modelos de Schumacher y Hossfeld IV son tan flexibles que además de sus familias de diferencia algebraica (DA) anamórficas o polimórficas pueden generar familias de diferencia algebraica generalizada (DAG), mismas que representan hipótesis más flexibles del patrón de crecimiento en los que tanto la asíntota como la tasa de crecimiento pueden variar con el sitio.

La familia anamórfica de DA para el modelo Schumacher es:

$$
A_{p}=A_{I} \times \exp \left(\beta_{1}\left(1 / E_{I}-1 / E_{p}\right)\right)
$$

Schumacher anamórfico

A partir de $\beta_{1}$ se deriva la familia polimórfica:

$A_{p}=\beta_{0}\left(\frac{A_{I}}{\beta_{0}}\right)^{\left(\frac{E_{I}}{E_{p}}\right)}$

Schumacher polimórfico

(Ec. 4)

Con el modelo Hossfeld IV es posible obtener tres familias de curvas. La anamórfica es de la forma:

$$
A_{p}=A_{I}\left(\frac{1+\exp \left(\alpha_{1}-\alpha_{2} \times \ln E_{I}\right)}{1+\exp \left(\alpha_{1}-\alpha_{2} \times \ln E_{P}\right)}\right)
$$

Hossfeld IV anamórfico

La familia polimórfica 1 derivada a partir de $\alpha_{1}$ es:

$\left.A_{p}=\alpha_{0} / 1+\left(\left(\frac{\alpha_{0}}{A_{I}}-1\right) \times\left(\frac{E_{I}}{E_{P}}\right)^{\alpha_{2}}\right)\right]$

Hossfeld IV polimórfico 1 
Finalmente, la familia polimórfica 2 derivada de $\alpha_{2}$ es:

$$
\left.A_{p}=\alpha_{0} / 1+\alpha_{1}\left(\frac{\alpha_{0}-A_{I}}{A_{I} \times \alpha_{1}}\right)^{\frac{\ln \left(E_{p}\right)}{\ln \left(E_{I}\right)}}\right]
$$

Hossfeld IV polimórfico 2

Las cinco formas presentadas (Ecs. 3 a 7) se usaron para definir la mejor opción de familia de curvas de IS para las especies en estudio. En este caso no se planteó aplicar modelos de DAG (Cieszewski y Bailey, 2000), ya que durante el proceso de su construcción uno de los parámetros específicos de sitio sufre un escalamiento que no es posible estimar, a menos que sólo se usen datos con remediciones. Para ajustar los Modelos 1 y 2 se emplearon mínimos cuadrados ordinarios no lineales, y se incorporó el efecto de la especie a través de variables indicadoras (dicotómicas).

\section{Criterio de validación}

Con los datos de las remediciones tomadas en 2006 se calcularon dos indicadores de predictibilidad de los modelos, el sesgo promedio y el factor de ajuste (Fang y Bailey, 1999). El sesgo promedio se define como:

$$
B_{\text {PROM }}=\frac{\sum_{j=1}^{K}\left(A_{j}\left(E_{R}\right)-\hat{A}_{j}\left(A_{0}, E_{0}, E_{R}\right)\right)^{2}}{K-p}
$$

Donde $A_{j}\left(E_{R}\right)$ es la altura dominante medida en la j-ésima parcela en 2006 a la edad de remedición $E_{R}$; $\hat{A}_{j}\left(A_{0}, E_{0}, E_{R}\right)$ es la altura proyectada en la j-ésima como referencia a la primera medición definida por $A_{0}$ y $E_{0} ; K$ es el número total de parcelas con remediciones; y $p$ es el número total de parámetros estimados estadísticamente en el modelo.

El factor de ajuste (FA) se definió como:

$$
F A=1-\frac{\sum_{j=1}^{K}\left(A_{j}\left(E_{R}\right)-\hat{A}_{j}\left(A_{0}, E_{0}, E_{R}\right)\right)^{2}}{\sum_{j=1}^{K}\left(A_{j}\left(E_{R}\right)-\bar{A}_{j}\left(E_{R}\right)\right)^{2}}
$$

donde $\bar{A}_{j}\left(E_{R}\right)$ es la media de alturas dominantes a la edad de remedición $E_{R}$.

Ambos criterios permiten visualizar la magnitud del error promedio así como el nivel de ajuste de manera porcentual. Se aplicaron para evaluar a los modelos tanto en el futuro como en un estado previo (denominado "retracción”). La validación al futuro toma como base a la altura dominante y a la edad medida en 2004-2005, y se compara este dato con la altura dominante observada en las parcelas remedidas en 2006. La "retracción" sucede cuando se usan los datos de la remedición del 2006 como referencia y se estima la altura dominante al momento del establecimiento de las parcelas en 2004-2005. Con esto se busca añadir una base más amplia para la selección de las familias de IS, ya que la utilidad práctica de estos modelos es poder estimar cualquier altura dominante a cualquier edad cuando se cuenta con una referencia inicial.

\section{RESULTADOS Y DISCUSIÓN}

\section{Ajuste $\mathbf{y}$ familias}

Los Modelos 1 y 2 se reescribieron para considerar el efecto de la especie; así, el Modelo 1 de Schumacher es:

$$
A_{i}=\left(\hat{\beta}_{0}+\hat{\beta}_{0 u} I_{u}\right) \times \exp \left(-\frac{\left(\hat{\beta}_{1}+\hat{\beta}_{1 u} I_{u}\right)}{E_{i}}\right)+\varepsilon_{i}
$$

Donde: $\hat{\beta}_{0 u}$ y $\hat{\beta}_{1 u}$ son los valores estimados de los parámetros asociados con Eucalyptus urophylla que se adicionan a los parámetros globales $\hat{\beta}_{0}$ y $\hat{\beta}_{1}$, los cuales describen el crecimiento de $E$. grandis; $I_{u}=\left\{\begin{array}{ll}1 & \text { E.urophylla } \\ 0 & \text { de otra manera }\end{array} ; E i\right.$ es el error de la $i$-ésima observación en la $A i$ altura medida a la edad $E i$.

El modelo de Hossfeld IV se reescribió como:

$$
A_{i}=\alpha_{0} \times\left(\begin{array}{l}
1+\exp \left(\hat{\alpha}_{1}+\hat{\alpha}_{1 u} I_{u}\right) \\
\times \exp \left(-\left(\alpha_{2}+\alpha_{2 u} I_{u}\right) \times \ln \left(E_{i}\right)\right)
\end{array}\right)^{-1}+\varepsilon_{i}
$$

Donde: $\hat{\alpha}_{1 u}$ y $\hat{\alpha}_{2 u}$ son los valores estimados de los parámetros asociados a Eucalyptus urophylla que se adicionan a los parámetros globales $\hat{\alpha}_{0}$ y $\hat{\alpha}_{1}$ que describen el crecimiento de $E$. grandis; las demás variables se definieron anteriormente.

Mientras que para el Modelo 8 todos los parámetros son estimables a través de regresión (Cuadro 1), para el Modelo 9 esto no es posible con los datos disponibles. Una 
razón es el bajo número de datos en edades de menos de 30 meses. Esta sub-representación hace que el ajuste del Modelo 9 tenga una varianza inflada y que los parámetros se vuelvan estadísticamente no significativos. Para solventar este problema la asíntota $\alpha_{0}$ se fijó en $40 \mathrm{~m}$, lo que garantiza un techo considerable para las familias de curvas.

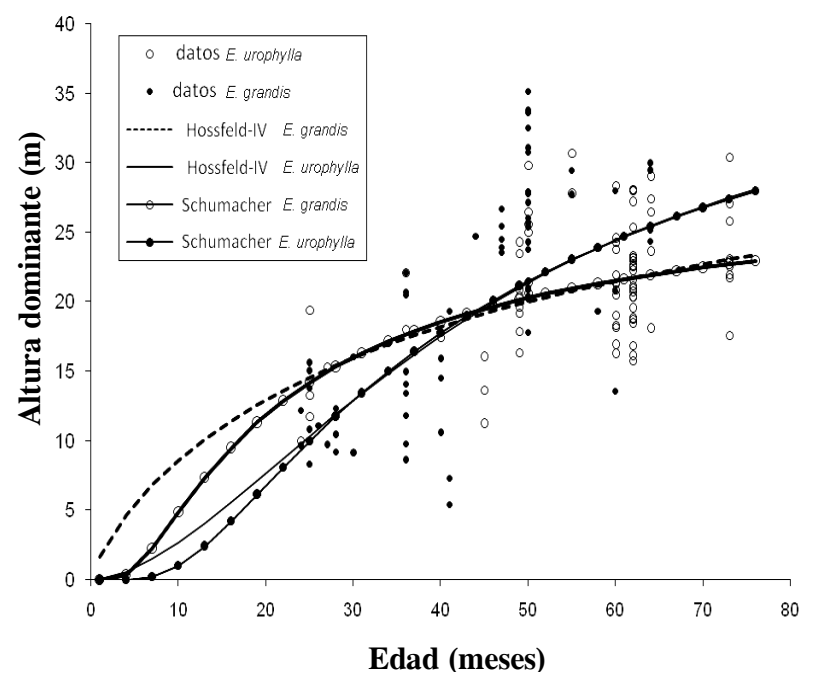

Figura 2. Curvas promedio ajustadas para los modelos de Schumacher (Ec. 8) y Hossfeld IV (Ec 9).

Para fijar este valor se tomó en cuenta lo siguiente: 1) Se asumió como constante al parámetro $\alpha_{0}$ que es el más no lineal y con mayores varianzas; 2) Para el Modelo 8 las asíntotas posibles sugeridas por el ajuste fueron de 29 y 46 $\mathrm{m}$, por lo que en varios ensayos con valores entre 30 y 45 m para $\alpha_{0}$ se encontraron que valores cercanos a 40 producían los mejores ajustes totales, y el resto de los parámetros fue altamente significativo; 3) Los datos graficados (Figura 1) sugieren que alturas mayores de 40 $\mathrm{m}$ son poco probables, por lo que establecer este valor como techo para rodales maduros es plausible, sobre todo por la tendencias en los sitios de mayor edad donde las ganancias en altura dominante son marginales. Como estas plantaciones son de rápido crecimiento, es poco probable que existan sitios con edades de más de 10 años, ya que generalmente se cosechan antes de alcanzar esa edad.

El ajuste de los Modelos 8 y 9 se presenta en el Cuadro 1 , donde se aprecia que ambos modelos tuvieron ajustes globales muy similares a los datos observados. Todos los parámetros fueron altamente significativos, de acuerdo con los valores de rechazo estimados. Es posible que cada especie pueda ser modelada de mejor manera por una curva en particular, y que no necesariamente ambas se ajusten al mismo modelo. Para probar esta hipótesis se combinaron alternadamente Schumacher 1 y Hossfeld IV 2 en un sólo ajuste. Inicialmente se planteó el caso donde E. grandis se ajusta bajo Schumacher y E. urophylla bajo Hossfeld IV, y se obtuvo un modelo de la forma:

$$
\begin{aligned}
& A_{i}=\left(\hat{\beta}_{0} \times \exp \left(-\frac{\beta_{1}}{E_{i}}\right)\right)^{1-I_{u}} \\
& \times\left(\alpha_{0} \times\left(\begin{array}{l}
1+\exp \left(\hat{\alpha}_{1 u}\right) \\
\left.\left.\left.\times \exp \left(-\left(\hat{\alpha}_{2 u}\right) \times \ln \left(E_{i}\right)\right)\right)\right)^{-1}\right)^{I_{u}}+\varepsilon_{i}
\end{array}\right.\right.
\end{aligned}
$$

En un segundo caso E. grandis se ajustó bajo Hossfeld IV y $E$. urophylla se ajustó bajo Schumacher y fue:

$$
\begin{aligned}
& A_{i}=\left(\beta_{0 u} \times \exp \left(-\frac{\beta_{1 u}}{E_{i}}\right)\right)^{I_{u}} \\
& \times\left(\alpha_{0} \times\left(\begin{array}{l}
1+\exp \left(\hat{\alpha}_{1}\right) \\
\left.\left.\times \exp \left(-\left(\hat{\alpha}_{2}\right) \times \ln \left(E_{i}\right)\right)\right)^{-1}\right)^{1-I_{u}}+\varepsilon_{i}
\end{array}\right.\right.
\end{aligned}
$$

A pesar de que los Modelos 10 y 11 no mejoraron el ajuste global de manera notable, y las ganancias resultaron muy marginales (Cuadro 1), los errores estándar de los parámetros resultaron menores, lo que redujo el valor de rechazo. Esto se atribuye al uso de un modelo que reduce la correlación entre parámetros y la escala de los mismos. 
Cuadro 1. Parámetros estimados y bondad de ajuste para los modelos de Schumacher (8), Hossfeld IV (9), y los modelos combinados por especie Schumacher-Hossfeld IV (10 y 11).

\begin{tabular}{|c|c|c|c|c|c|c|}
\hline Ecuación & GL Modelo & $\begin{array}{c}\mathrm{GL} \\
\text { Error }\end{array}$ & SCE & CME & $\begin{array}{l}\text { Raíz } \\
\text { CME }\end{array}$ & $\mathrm{R}^{2}$ \\
\hline 8 & 4 & 149 & 2879.5 & 19.3256 & 4.3961 & 0.4814 \\
\hline 9 & 4 & 149 & 2856.0 & 19.1679 & 4.3781 & 0.4753 \\
\hline 10 & 4 & 149 & 2876.4 & 19.3045 & 4.3937 & 0.4820 \\
\hline 11 & 4 & 149 & 2859.2 & 19.1890 & 4.3805 & 0.4851 \\
\hline \multirow{5}{*}{$\begin{array}{c}\text { Ecuación } \\
8\end{array}$} & Parámetro & & Estimado & $\begin{array}{c}\text { Error } \\
\text { Estándar }\end{array}$ & $\begin{array}{c}\text { 't' } \\
\text { calculada }\end{array}$ & $\operatorname{Prob}>|\mathbf{t}|$ \\
\hline & $\beta_{0}$ & & 29.00 & 2.56 & 11.31 & $<.0001$ \\
\hline & $\beta_{0 u}$ & & 17.29 & 4.91 & 3.52 & 0.0006 \\
\hline & $\beta_{1}$ & & 17.87 & 4.86 & 3.68 & 0.0003 \\
\hline & $\beta_{1 u}$ & & 20.48 & 6.42 & 3.19 & 0.0017 \\
\hline \multirow[t]{5}{*}{9} & $\alpha_{0}$ & & 40 & - & - & - \\
\hline & $\alpha_{1}$ & & 3.16 & 0.79 & 3.99 & 0.0001 \\
\hline & $\alpha_{1 u}$ & & 3.43 & 1.04 & 3.3 & 0.0012 \\
\hline & $\alpha_{2}$ & & 0.81 & 0.19 & 4.12 & $<.0001$ \\
\hline & $\alpha_{2 u}$ & & 0.91 & 0.26 & 3.45 & 0.0007 \\
\hline \multirow[t]{5}{*}{10} & $\alpha_{0}$ & & 40 & - & - & - \\
\hline & $\alpha_{1 u}$ & & 3.16 & 0.79 & 3.98 & 0.0001 \\
\hline & $\alpha_{2 u}$ & & 0.81 & 0.19 & 4.10 & $<.0001$ \\
\hline & $\beta_{0}$ & & 46.32 & 4.19 & 11.06 & $<.0001$ \\
\hline & $\beta_{1}$ & & 38.39 & 4.19 & 9.16 & $<.0001$ \\
\hline \multirow[t]{6}{*}{11} & $\alpha_{0}$ & & 40 & - & - & - \\
\hline & $\alpha_{1}$ & & 6.59 & 0.67 & 9.81 & $<.0001$ \\
\hline & $\alpha_{2}$ & & 1.72 & 0.18 & 9.72 & $<.0001$ \\
\hline & $\alpha_{2}$ & & 1.72 & 0.18 & 9.72 & $<.0001$ \\
\hline & $\beta_{0 u}$ & & 29.00 & 2.55 & 11.35 & $<.0001$ \\
\hline & $\beta_{1 u}$ & & 17.88 & 4.85 & 3.69 & 0.0003 \\
\hline
\end{tabular}

$\mathrm{GL}=$ Grados de libertad; SCE $=$ Suma de cuadrados del error; CME $=$ Cuadrado medio del error; $\mathrm{R}^{2}=$ Coeficiente de determinación del modelo.

Los parámetros estimados por especie no difieren de los producidos por el ajuste de los Modelos 8 y 9, que fueron los utilizados para la validación y proyección de las familias.

\section{Validación y definición de familias}

Para la validación se decidió primero evaluar las familias en su comportamiento global al suponer la misma familia para ambas especies (Cuadro 2). Con el sesgo promedio y el factor de ajuste (FA) se calificaron los modelos en forma ascendente y se otorgó la calificación más baja a la mejor familia en las proyecciones y retracciones. Para obtener una calificación total de la predictibilidad se sumaron las calificaciones por familia. Así, tanto para las proyecciones como para las retracciones, el mejor modelo es el polimórfico 1 de Hossfeld IV (Ec. 6) que dio la calificación total (CT) más baja. El segundo lugar lo ocupa la familia anamórfica de Hossfeld IV (Ec 5). Las familias de Schumacher son poco aptas en términos del ajuste total, al igual que la familia polimórfica 1 de Hossfeld IV, por dar las CT más altas.

Al considerar que se requiere más flexibilidad para calificar la capacidad de predicción, se validaron las proyecciones y retracciones por familia de curvas y por especie (Cuadro 3). De la misma manera que en el caso general descrito, se calificaron los modelos por especie y se calcularon las calificaciones para cada caso y la total por especie. Se encontró que para $E$. grandis las familias anamórfica y polimórfica 1 de Hossfeld IV fueron las que ofrecieron la menor CT de las cinco familias posibles. 
Cuadro 2. Sesgo promedio, factor de ajuste (FA), calificaciones por grupo (CP) y totales (CT) para las proyecciones y retracciones de las familias de índice de sitio de Schumacher y Hossfeld IV.

\begin{tabular}{cccccc}
\hline $\begin{array}{c}\text { Esquema de } \\
\text { Validación }\end{array}$ & Familia de curvas & $\begin{array}{c}\text { Sesgo } \\
\text { promedio }\end{array}$ & FA & CP & CT \\
\hline Proyección & Schumacher anamórfico & 6.69 & 0.68 & 5 & 8 \\
& Schumacher polimórfico & 2.59 & 0.88 & 2 & 7 \\
& Hossfeld IV anamórfica & 5.61 & 0.73 & 3 & 5 \\
& Hossfeld IV polimórfica 1 & 2.09 & 0.90 & 1 & 2 \\
& Hossfeld IV polimórfica 2 & 6.37 & 0.70 & 4 & 8 \\
& & & & & \\
& Schumacher anamórfico & 2.55 & 0.76 & 3 & - \\
Retracción & Schumacher polimórfico & 3.85 & 0.63 & 5 & - \\
& Hossfeld IV anamórfica & 3.19 & 0.85 & 2 & - \\
& Hossfeld IV polimórfica 1 & 2.17 & 0.90 & 1 & - \\
& Hossfeld IV polimórfica 2 & 5.43 & 0.74 & 4 & - \\
\hline
\end{tabular}

Cuadro 3. Sesgo promedio, factor de ajuste (FA), calificaciones por familia por especie (CFE) y totales (CTE) para las proyecciones y retracciones de las familias de IS de Schumacher y Hossfeld IV.

\begin{tabular}{|c|c|c|c|c|c|c|c|c|c|}
\hline \multirow[b]{2}{*}{ Especie } & \multirow[b]{2}{*}{ Modelo } & \multirow[b]{2}{*}{ Familia } & \multicolumn{3}{|c|}{ Proyección } & \multicolumn{3}{|c|}{ Retracción } & \multirow[b]{2}{*}{ CTE } \\
\hline & & & Sesgo & FA & CFE & Sesgo & FA & CFE & \\
\hline \multirow{6}{*}{ E. grandis } & \multirow[t]{2}{*}{ Schumacher } & Anamórfica & 2.82 & 0.73 & 4 & 2.55 & 0.76 & 3 & 7 \\
\hline & & Polimórfica & 2.77 & 0.74 & 3 & 3.85 & 0.63 & 4 & 7 \\
\hline & & & & & & & & & \\
\hline & \multirow[t]{3}{*}{ Hossfeld IV } & Anamórfica & 2.00 & 0.81 & 2 & 1.74 & 0.83 & 1 & 3 \\
\hline & & Polimórfica 1 & 1.93 & 0.82 & 1 & 2.05 & 0.80 & 2 & 3 \\
\hline & & Polimórfica 2 & 4.48 & 0.57 & 5 & 4.16 & 0.60 & 5 & 10 \\
\hline \multirow{6}{*}{ E. urophylla } & \multirow[t]{2}{*}{ Schumacher } & Anamórfica & 10.10 & 0.66 & 5 & 4.83 & 0.84 & 4 & 9 \\
\hline & & Polimórfica & 2.42 & 0.92 & 2 & 2.51 & 0.92 & 2 & 4 \\
\hline & & & & & & & & & \\
\hline & \multirow[t]{3}{*}{ Hossfeld IV } & Anamórfica & 8.79 & 0.71 & 4 & 4.46 & 0.85 & 3 & 7 \\
\hline & & Polimórfica 1 & 2.23 & 0.93 & 1 & 2.27 & 0.92 & 1 & 2 \\
\hline & & Polimórfica 2 & 8.05 & 0.73 & 3 & 6.54 & 0.78 & 5 & 8 \\
\hline
\end{tabular}

Aunque ambas tienen la misma $\mathrm{CT}=3$, el sesgo medio entre proyecciones y retracciones de la familia anamórfica Hossfeld IV (1.86) es menor que el de la polimórfica 1 (1.99). El factor de ajuste medio entre proyecciones y retracciones es respectivamente 0.822 y 0.810 . Ambas familias se muestran en la Figura 3 bajo cuatro condiciones de IS.

Para E. urophylla la familia de curvas con la mejor calificación total es la polimórfica 1 de Hossfeld IV, tanto para proyectar como para retraer, con una $\mathrm{CT}=2$, el valor mínimo posible. En segundo lugar se identificó a la familia polimórfica de Schumacher con una CT $=4$. Para esta especie los patrones polimórficos fueron los que mejor describieron el patrón de crecimiento en altura dominante. Ambas proyecciones se muestran en la Figura 4.
En las Figuras 3 y 4 se presentan las mejores familias de IS, en donde puede apreciarse que para $E$. grandis la familia anamórfica de Hosfeld IV produjo excelentes proyecciones para edades mayores de 50 meses, pero es conservadora respecto a las estimaciones de la familia polimórfica 1 de Hosfeld IV en edades jóvenes (una proyección conservadora es la que a la misma edad produce menores estimaciones de alturas dominantes). De forma similar, la familia polimórfica 1 de Hosfeld IV se ajustó a los datos de más de 50 meses de edad, pero es muy optimista en las mejores calidades, comportamiento que no puede descartarse si se observa que se tiene una parcela remedida (ver en Figura 3 una parcela de remedición cuya altura a los 30 meses es de $25 \mathrm{~m}$ ) que sugiere este comportamiento. 


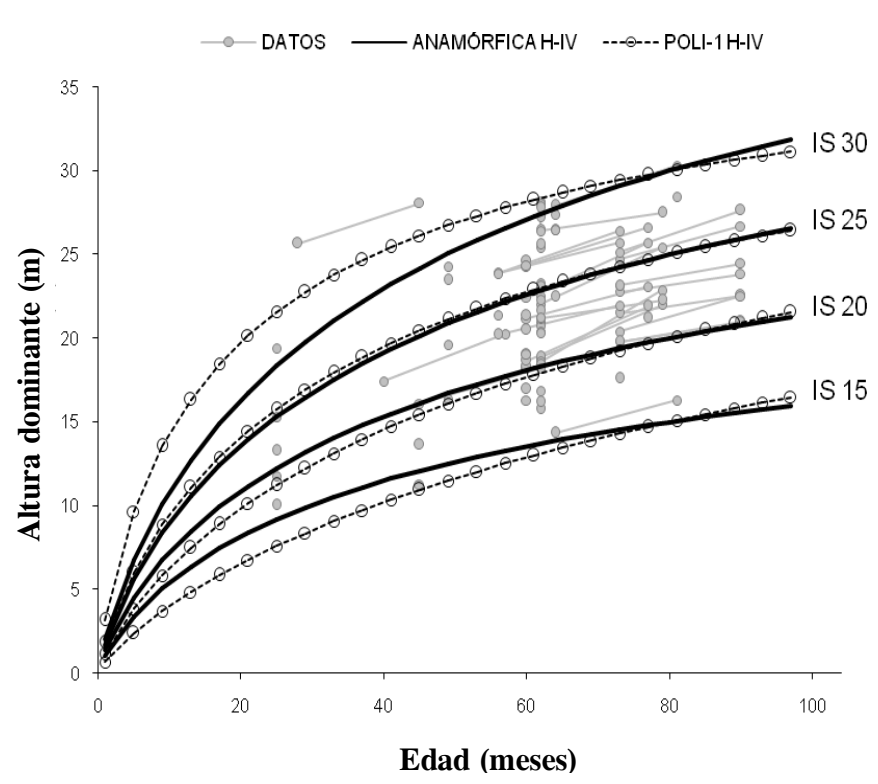

Figura 3. Familias de índice de sitio de 15, 20, 25 y $30 \mathrm{~m}$ a la edad base de 80 meses. Familias anamórfica (ANAMÓRFICA H-IV) y polimórfica 1 de Hossfeld IV (POLI -1 H-IV) en Eucalyptus grandis.

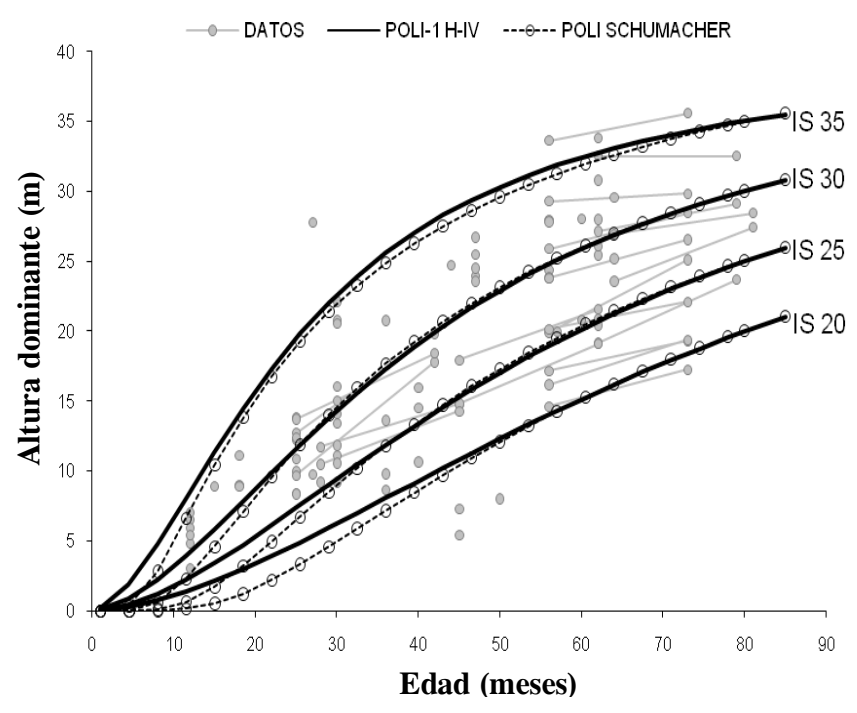

Figura 4. Familias de índice de sitio de 20, 25, 30 y $35 \mathrm{~m}$ a la edad base de 80 meses para las familias polimórfica 1 de Hossfeld IV (POLI-1 H-IV) y polimórfica de Schumacher (POLI SCHUMCHER) en Eucalyptus urophylla.

Para E. urophylla las dos familias de curvas polimórficas ajustadas (Figura 4) mostraron características deseables de patrones de crecimiento, al comparar visualmente la dispersión de datos. En este caso la familia polimórfica de Schumacher es conservadora a edades jóvenes comparada con la curva Polimórfica 1 de Hossfeld IV.
El criterio de validación-selección empírico propuesto permitió establecer una línea base cuantitativa para la selección de las familias de IS, condición que no es posible definir cuando se utilizan criterios como el ajuste global de la curva guía. Sin este criterio, la decisión respecto a las mejores familias se habría tomado con base en un análisis visual. Métodos alternativos como la validación cruzada, son más adecuados cuando se busca calificar a los modelos con base en sus condiciones de ajuste paramétrico (Kozak y Kozak, 2003).

\section{Uso de los modelos (familias)}

El grupo de familias mostradas en las Figuras 3 y 4 podrían utilizarse para definir y representar tres zonas equiproductivas representativas del área de estudio, y los sitios más aptos para $E$. grandis serán aquellos cuyo IS es mayor de $25 \mathrm{~m}$ con una edad base de 80 meses; los sitos medios serían aquellos con IS mayor de 20 pero menor de 25; y los sitios pobres los que tengan un IS menor de 20. Esto resultaría así en el supuesto de que se desee tener el área total de las plantaciones divida en tres categorías de IS para esta especie. También es posible generar mapas continuos de productividad a partir del cálculo puntual del IS y de una red de sitios de inventario geo-referenciados, mediante la interpolación de la información de productividad. Todo depende del tipo de información que el manejador del área necesite. Por ejemplo, Telles et al. (2008) definieron tres clases de IS por especie para realizar un estudio financiero en los predios de PLANTEH, lo que simplificó el análisis y permitió elaborar corridas financieras por cada clase de IS.

En caso de que las plantaciones decidieran manejarse después de la primera cosecha como monte bajo (rebrotes de tocones), es necesario calibrar o construir los modelos de IS que coincidan con estas condiciones; de igual manera en caso de que se decida usar clones.

Los métodos utilizados son resultado de las características propias de los datos tomados en el estudio donde no se tienen remediciones de todos los sitios. Para obtener mejores resultados en el ajuste, validación y descripción de los patrones de altura se requieren observaciones múltiples por parcelas (al menos dos puntos en el tiempo) para estimar de manera simultánea los elementos comunes de los vectores $p$ y $p^{(g)}$ para $A_{2}=g\left(E_{1}, E_{2}, A_{1} ; p^{(g)}\right)$ y $A_{1}=f\left(E_{1} ; p\right)$, para lograr así las siguientes ventajas: 1) Compatibilidad absoluta entre predicción y proyección; y 2) Mejores características de los parámetros estimados, como una mayor eficiencia estadística y menor sesgo (Borders, 1989; Galan et al., 2008). Con este tipo de datos es posible aislar el efecto del 
sitio vía modelos de efectos mixtos (Calegario et al., 2005), que se considera el método de ajuste estadístico más adecuado para datos de naturaleza longitudinal, como los datos de crecimiento en altura (mediante observaciones sucesivas a través del tiempo).

\section{CONCLUSIONES}

Para las plantaciones evaluadas se propone el siguiente sistema de índice de sitio en función de la edad. Para Eucalyptus grandis el modelo más adecuado es la familia anamórfica de Hossfeld V de la forma:

$$
A_{p}=A_{I}\left(\frac{1+\exp \left(3.16-0.81 \times \ln E_{I}\right)}{1+\exp \left(3.16-0.81 \times \ln E_{P}\right)}\right)
$$

Mientras que para E. urophylla el modelo de IS más adecuado está dado por la familia polimórfica 1 de Hossfeld IV de la forma:

$$
A_{p}=\frac{40}{1+\left(\left(\frac{40}{A_{I}}-1\right) \times\left(\frac{E_{I}}{E_{P}}\right)^{1.72}\right)}
$$

Los modelos descritos podrían usarse en el manejo forestal en las plantaciones provenientes de semilla de la región de estudio.

\section{AGRADECIMIENTOS}

Al Consejo Nacional de Ciencia y Tecnología y Comisión Nacional Forestal por el financiamiento de esta investigación mediante el proyecto CONAFOR 2003-CO39925. A la empresa Plantaciones de Tehuantepec S. A. de C. V., por el apoyo brindado para efectuar esta investigación.

\section{BIBLIOGRAFÍA}

Amaro A, D Reed, T Margarida, I Themido (1998) Modeling dominant height growth: Eucalyptus plantations in Portugal. For. Sci. 44:37-46.

Borders B E (1989) Systems of equations in forest modeling. For. Sci. 35:548-556.

Calegario N, R F Daniels, R Maestri, R Neiva (2005) Modeling dominant height growth based on nonlinear mixed-effects model: a clonal Eucalyptus plantation case study. For. Ecol. Manage. 204:11-20

Cieszewski C J (2001) Comparing fixed-and variable- base-age site equations having single versus multiple asymptotes. For. Sci. 48:7-23.

Cieszewski C J (2003) Developing a Well-Behaved dynamic site equation using a modified Hossfeld IV function $\mathrm{Y}^{3}=\left(\mathrm{ax}^{\mathrm{m}}\right) /\left(\mathrm{c}+\mathrm{x}^{\mathrm{m}-1}\right)$, a simplified mixed-model and scant subalpine fir data. For. Sci. 49:539-554

Cieszewski C J, R L Bailey (2000) Generalized algebraic difference approach: theory based derivation of dynamic site equations with polymorphism and variable asymptotes. For. Sci. 46:116-126.

Fang X, R L Bailey (1999) Compatible volume and taper model with coefficients for tropical species on the Hainan Island in southern China. For. Sci. 45:85-100.

FAO/UNESCO (1970) Clave de Unidades de Suelos para el Mapa de Suelos del Mundo. Secretaria de Recursos Hidráulicos. Dirección General de Estudios. Dirección de Agroecología. México, D. F.

Galán L R, H M De los Santos P, J I Valdez H (2008) Crecimiento y rendimiento maderable de Cedrela odorata L. y Tabebuia donnell-smithii Rose en San José Chacalapa, Pochutla, Oaxaca. Madera y Bosques 14:65-82.

García M E (1988) Modificaciones al Sistema de Clasificación Climática de Köppen, para Adaptarlo a las Condiciones de la República Mexicana. Instituto de Geografía. UNAM. México, D. F. 243 p.

Kiviste A J, R A Álvarez-Alboreca, A D $\mathbf{R}$ González (2002) Funciones de crecimiento en el ámbito forestal. Comunicaciones INIA S. Rec. Nat. 4.75 p.

Kozak A, R Kozak (2003) Does cross validation provide additional information in the evaluation of regression models? Can. J. For. Res. 33:976-987.

Schumacher F X (1939) A new growth curve and its applications to timber yield studies. J. For. 37:819-820.

Telles M E, M J González G, H M De los Santos P, A M Fierros G, R J Lilieholm, A Gómez G (2008) Rotación óptima en plantaciones de eucalipto al incluir ingresos por captura de carbono en Oaxaca, México. Rev. Fitotec. Mex. 31:173-182.

Wang Y, V M LeMay, T G Baker (2007) Modeling and prediction of dominant height and site index of Eucalyptus globules plantations using a non-linear mixed effects model approach. Can. J. For. Res. 37:1390-1403.

Zeide B (1993) Analysis of growth equations. For. Sci. 39:594-616. 